\title{
Sensorless Active Damping Strategy for Parallel Interleaved Voltage Source Power Converters with LCL Filter
}

\author{
Javier Samanes, Student Member, IEEE and Eugenio Gubía, Member, IEEE \\ Department of Electrical and Electronic Engineering - Institute of Smart Cities \\ Public University of Navarre \\ Pamplona, Spain \\ javier.samanes@unavarra.es
}

\begin{abstract}
Grid-connected pulse-width modulation converters with LCL filters are widely extended as an interface for renewable energy generating systems. Those filters arise stability issues due to the filter resonance, which, must be damped, preferably by active damping methods, to avoid additional power losses. In some applications, such as offshore wind energy conversion systems, where high power converters are connected to low voltage networks, the commutation frequency is limited to low values to reduce the power losses and the resulting filter components are bulky. For this reason, in addition to its higher current handling capabilities, the use of power converters in parallel with the application of advanced modulation strategies, such as interleaving, is gaining importance. In this structure the filter components can be reduced, setting the filter resonance frequency at a higher value. As a consequence, the filter resonance frequency is close to the Nyquist frequency, complicating the implementation of some of the existing active damping solutions. In this work, an active damping approach based on a third order digital filter is proposed to stabilize the system. The robustness of the solution is investigated against grid inductance variations and the grid current harmonic content will be tested to comply with the most demanding grid codes. The proposed active damping strategy does not require extra sensors and can be easily designed by examining the open loop Bode plots. It is suitable to stabilize the LCL filter with a resonance frequency close to the Nyquist frequency, where some of the existing active damping approaches are unable to damp the system. The proposed method can be also an appropriate solution for existing grid connected power converters with stability issues due to changes in the effective grid impedance at which they are connected.
\end{abstract}

Keywords-Active damping; LCL filter; stability; grid-connected pulse-width modulation (PWM) converters; three phase voltage source converters (VSC); parallel interleaved power converters.

\section{I.INTRODUCTION}

Grid-connected pulse-width modulation converters are widely extended, as an interface for renewable energy generating systems. As a consequence of the increasing number of power converters connected to the grid, stringent grid codes have been developed in order to limit the current harmonic content at the point of common coupling (PCC) [1]-[3]. To fulfill those grid codes a passive filter at the output of the power converter is inserted. The use of LCL passive filters is a standard solution to limit the harmonic content, as they allow the reduction of the filter components with respect to the $\mathrm{L}$ filter, without penalizing its dynamic response [4]. The use of LCLfilters arises some concerns such as system instability that can be avoided by the passive or active damping of the filter [5].

In passive damping methods additional damping resistors are connected to the LCL filter, increasing its power losses. A direct and commonly used approach is the addition of a resistor in series with the filter capacitor. Other passive damping options present a better efficiency, but the number of passive components and the filter complexity is increased [6].

As an alternative, active damping approaches have been widely explored. By modifying the control loop, stability can be guaranteed. Current feedback of the filter capacitor is one of the most studied and extended options [5], [7]. Its main drawback is that additional current sensors are required. For this reason, as the voltage can be sensed at the capacitor for grid synchronization, the voltage feedback of the capacitor LCL filter has been considered [8]. Other options, without additional sensors, include the design of filters in the control path [9] and the introduction of delays in the control loop [10]. These active damping techniques are an appropriate solution for low and medium power applications, where the filter resonance frequency can be located far enough from the switching frequency. As the converter rated power is increased, and the switching frequency is limited to low values, to reduce the commutation power losses, the resonance frequency gets closer to the Nyquist frequency compromising the effectiveness of the digital implementation. Moreover, the active damping strategy must be robust to grid inductance variations, as the grid conditions may vary through the power converter life cycle.

Renewable energy generating systems, particularly wind turbines, are constantly incrementing their power ratings [11]. As the electric generators used in wind turbine are commonly low voltage (LV) generators, to escalate the power rating of the power converter several two-level three-phase LV VSC can be parallelized to increase their current handling capabilities. The application of advanced modulation techniques such as PWM carrier interleaving reduce the requirements on the filter [12], 

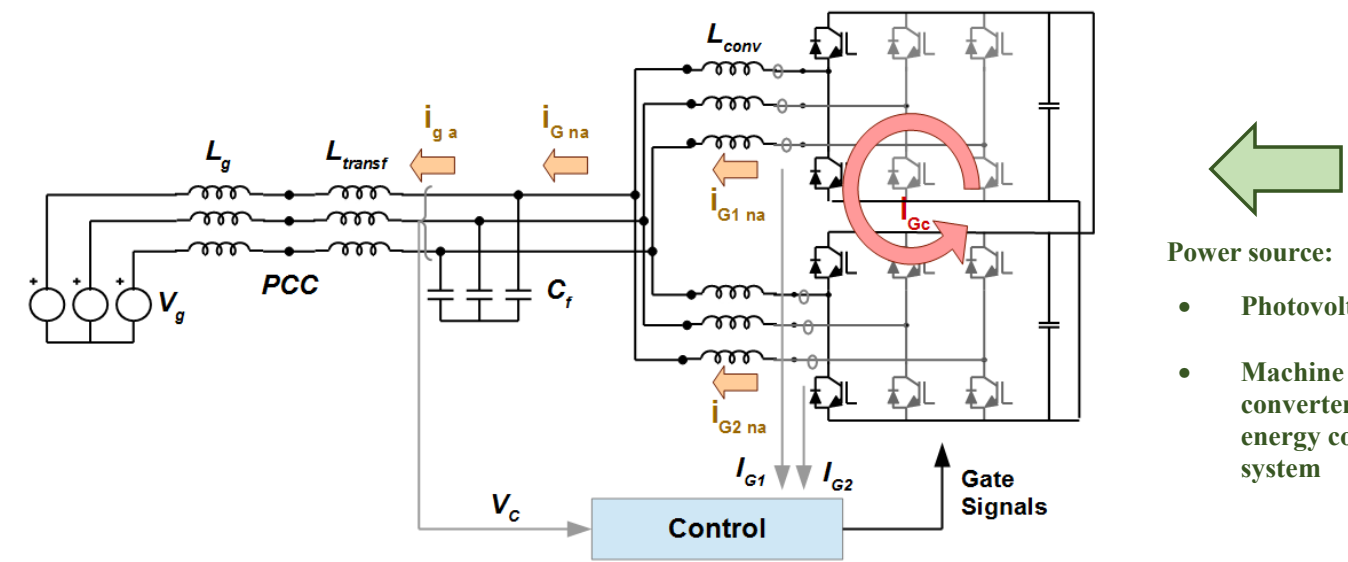

Power source:

- Photovoltaic system

- Machine side

converter of wind energy conversion system

Fig. 1. System under study.

[13] as it might be designed to attenuate higher order harmonics than the commutation frequency harmonics. The higher resonance frequency of the LCL filter strongly affects the digital implementation of the active damping compensators.

In this work a study of the digital implementation of the controllers and the system stability at frequencies close to the Nyquist frequency is developed. As a result of the analysis an active damping strategy for voltage source parallel power converters is developed and its performance is studied through simulation. The proposed approach does not require extra sensors and can be easily designed by examining the Bode plots.

In Section II, the system under study is presented and modeled. In Section III the most relevant active damping approaches are discussed, and in Section IV the filter based strategy developed in this work, is introduced. In Section V the simulation results are presented and the grid code compliance is evaluated. Finally, in Section VI the actively damped system is compared to the passively damped system in terms of efficiency, under the same design conditions.

\section{Modeling AND CONTROL OF THE Three Phase PARAllel INTERLEAVED VSC}

\section{A. System analysis and modeling of the grid connected power converter}

In wind power and photovoltaic systems, the grid connection of the power converter to the medium voltage grid is performed by a step up transformer, and thus the transformer leakage inductance, $L_{\text {transf, }}$ can be considered a part of the LCL filter, as depicted in Fig. 1. $L_{g}$ is the grid inductance at the point of common coupling (PCC) and depends on the grid at which the power converter is connected. $L_{c o n v}$ is the converter inductance and $C_{f}$ is the filter capacitor.

Each converter output current can be divided in the circulating current component, $i_{G c}$, recirculated between both power converters, and the normal current component injected to the grid, $i_{G x n}$, defined as half of the grid current [14], where $x$ stands for the converter number and $G$ for the grid side converters. The power source is not depicted. In Fig. 1, independent inductances have been considered, even though, interphase transformers can be used to limit the circulating currents and reduce the resulting LCL filter components [15], [16]. As the scope of the present paper is to study the resonance of the LCL grid filter, only the normal component of the current is taken into account.

The single phase equivalent model for the normal component of the two parallel power converters with the LCL filter is detailed in Fig. 2 (a). $V_{G x n i}$ is the normal component of the voltage modulated by the grid side converter $x$ for the phase $i$. As only the normal component is modeled, the equivalent circuit in Fig. 2 (b) can be derived, where $V_{G x n i}$ is the mean of $V_{G I n i}$ and $V_{G 2 n i}$. From this figure, the resonant frequency of the grid current to the converter output voltage is given by (1).

$$
f_{\text {res }}=\frac{1}{2 \pi} \sqrt{\frac{L_{\text {conv }} / 2+\left(L_{\text {transf }}+L_{g}\right)}{L_{\text {conv }} / 2\left(L_{\text {transf }}+L_{g}\right) C_{f}}}
$$

The filter components, $L_{\text {conv }}, L_{\text {transf }}$ and $C_{f}$ are designed to attenuate the high frequency switching ripple. In an interleaved power converter, consisting in two power converters coupled in parallel, harmonics at the switching frequency are 180 degrees phase shifted and thus recirculated [17], [18]. The LCL filter is then designed to attenuate the second commutation family. The filter parameters along with the converter specifications can be found in Table 1. The current normal component harmonics are limited by half of the converter inductance, the filter capacitor and the grid and transformer inductances. The current circulating harmonics are filtered by two times the converter filter inductance. If three phase inductors are used, the converter inductance does not filter the common mode cross current component, which, would raise unacceptable levels. For this reason, along with the reduction of the overall magnetic components, the use of IPTs is the preferred option.

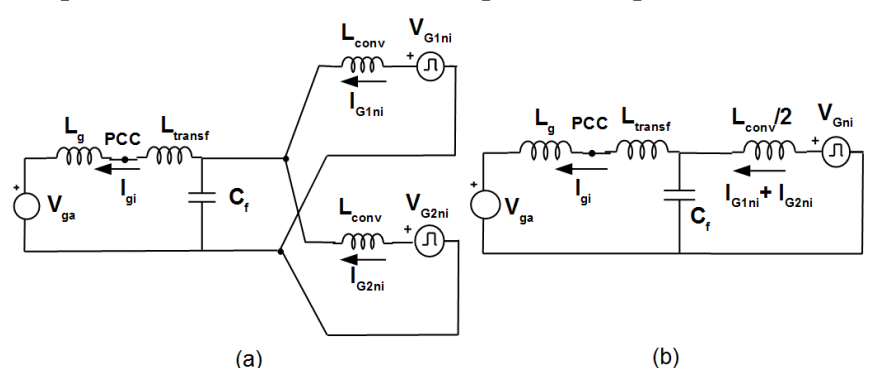

Fig.2. Single phase filter equivalent model 
TABLE I.

SYSTEM PARAMETERS

\begin{tabular}{|l|l|l|l|}
\hline \multicolumn{1}{|c|}{ Parameter } & \multicolumn{1}{c|}{ Value } & \multicolumn{1}{c|}{ Parameter } & \multicolumn{1}{c|}{ Value } \\
\hline $\mathrm{V}_{\mathrm{g}}$ & $690 \mathrm{~V}$ & $\mathrm{Cf}$ & $800 \mu \mathrm{F}$ \\
\hline $\mathrm{P}_{\text {nom }}$ & $4 \mathrm{MW}$ & $\mathrm{L}_{\mathrm{g}}$ & $1-200 \mu \mathrm{H}$ \\
\hline $\mathrm{F}_{\text {conm }} / \mathrm{F}_{\text {samp }}$ & $1950 / 3900 \mathrm{~Hz}$ & $\mathrm{~F}_{\text {res }}$ & $1600 / 1095 \mathrm{~Hz}$ \\
\cline { 1 - 3 } $\mathrm{L}_{\text {conv }} / \mathrm{L}_{\text {conv out }}$ & $60 \mu \mathrm{H} / 30 \mu \mathrm{H}$ & $\mathrm{F}_{\text {res }} / \mathrm{F}_{\mathrm{Ny}}$ & $0.83-0.53$ \\
\cline { 1 - 2 } $\mathrm{L}_{\text {transf }}$ & $20 \mu \mathrm{H}$ & \multicolumn{3}{|r}{} \\
\cline { 1 - 2 } & & &
\end{tabular}

The grid inductance seen by each power converter depends on the grid and the power connected at the PCC [19], for this reason, the filter resonance can change. Nevertheless, the resonance frequency variation has an asymptote whose value corresponds to the limit of (1) when $L_{g}$ tends to infinity (2), Fig. 3.

$$
f_{\text {res }}=\frac{1}{2 \pi} \sqrt{\frac{1}{L_{\text {conv }} / 2 C_{f}}}
$$

\section{B. Control strategy and representation of the digital controller}

In parallel power converters the converter side current is usually measured in order to be able to control the circulating currents, as shown in Fig. 1, and thus it is used for the grid current control loop. The converter normal component of the converter current can be controlled by means of a PI controller in the synchronous reference frame. The phase margin and bandwidth are set to 60 degrees and $120 \mathrm{~Hz}$, respectively.

As represented in Fig. 4, the control loop combines elements in the discrete time domain, such as the controller, with elements in the continuous time, such as the LCL filter and the PWM inverter.

In order to properly analyze the system stability, all the elements must be represented in the same domain. The continuous time domain is chosen for the representation as it offers a more intuitive approach. The analogical representation of the digital processing, a sample delay, and the PWM, approximated by a zero order hold, is modeled by $\mathrm{G}_{\mathrm{conv}}(\mathrm{s})(4)$, [19].

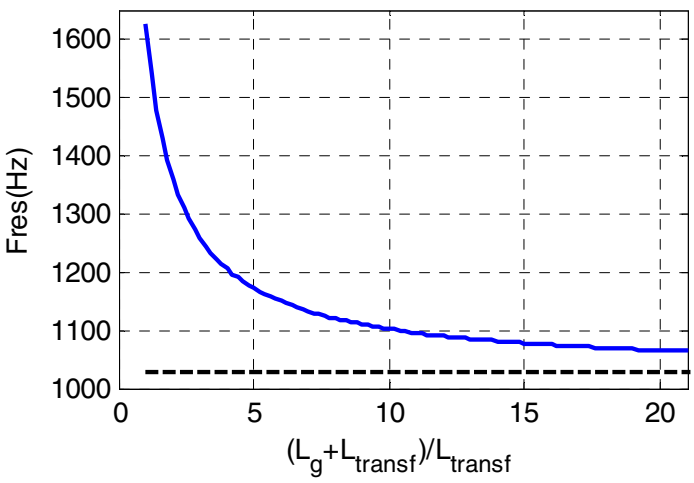

Fig. 3. Resonance frequency evolution as the grid inductance is increased.

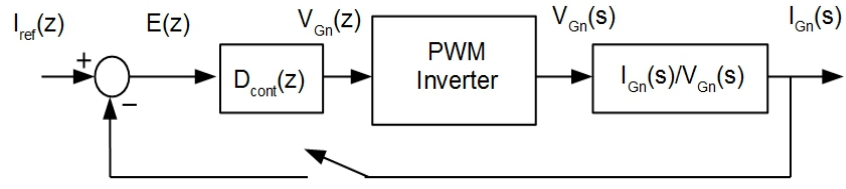

Fig.4. Current control loop

$$
G_{\text {conv }}(s)=\frac{e^{-T_{\text {samp }} s}\left(1-e^{-T_{\text {sanp }} s}\right)}{T_{\text {samp }} s}
$$

As a consequence of the application of interleaving between both power converters, the LCL filter is designed to attenuate the second harmonic family. Due to that, the filter resonance frequency is located close below the commutation frequency. As the asymmetrical regular sampling [20] creates a lower harmonic content, the sampling frequency is twice the commutation frequency. The resonance frequency is thus close to the Nyquist frequency, which is equal to the switching frequency. To evaluate the stability, an accurate approximation for the digitalization (4) must be used at frequencies, close to the Nyquist frequency.

Fig. 5 shows the frequency response of the expression in (4), blue line, along with the approximations commonly used in the literature to avoid the computational cost of the exact representation. Those approximations are not suitable for the purpose of this work. The first order approximation [21], green line, introduces a phase delay close to the Nyquist of $-90^{\circ}$. The second order approximation [19], in red, calculated by substitution of the Padé first order approximation of the delay introduces $-180^{\circ}$. As these expressions are far from the $-270^{\circ}$ degrees close to Nyquist frequency of the expression in (4), a fourth order approximation has been developed. Following the approach presented in [19] the Padé second order approximation for the continuous delay is substituted in (4), deriving the expression (5).

$$
G_{\text {cont }}(s) \approx \frac{12 T_{\text {samp }}{ }^{2} s^{2}-72 T_{\text {samp }} s+144}{T_{\text {samp }}{ }^{4} s^{4}+12 T_{\text {samp }} s^{3}+60 T_{\text {samp }} s^{2}+144 T_{\text {sam }} s+144}
$$
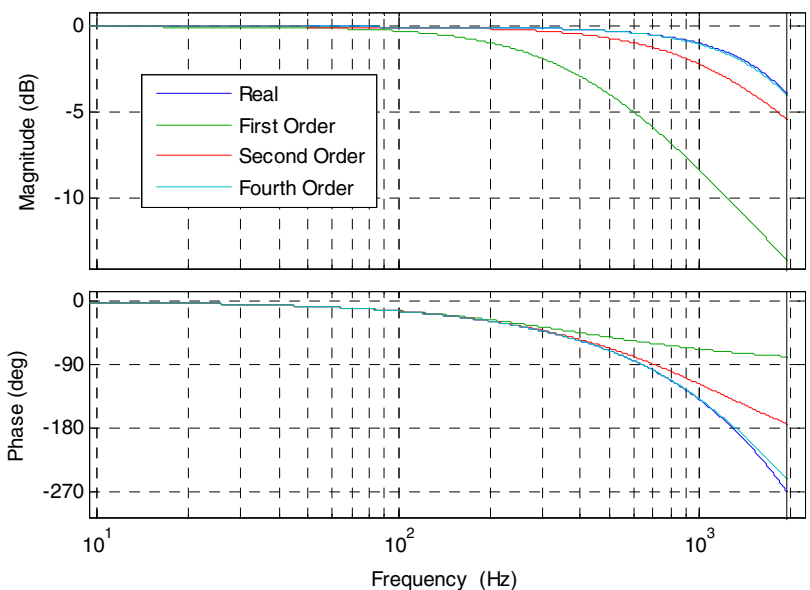

Fig. 5. Comparison of the different approximations for the digital controller emulator. 


\section{EVAluation of EXISTING Active DAMPING TeChNiQues}

The main goal of the active damping techniques is to guarantee the stable operation of the power converter. The Nyquist stability criteria is used to evaluate the system stability. To attain stability the number of positive crossings, from a phase lower than $\pm(2 \mathrm{k}-1) \times 180^{\circ}$ to a phase greater than $\pm(2 \mathrm{k}-1) \times 180^{\circ}$, where $\mathrm{k}$ is a positive integer, must equal the number of negative crossings as there are no unstable poles in the system. If stability is verified between the values given by Table I for $L_{g}$, the converter would be stable for every operating point.

In order to analyze the stability of the converter current loop, the open loop bode plots are represented in Fig. 6. Expression (5) is used to model the sample delay and the zero order hold. The PI controller is also included. Without additional damping actions the system is unstable for different grid inductance values. As depicted in Fig. 6, the system has a negative zero crossing, marked with a red spot, and no positive zero crossings, meaning that the closed loop transfer function has unstable poles. For this reason, an active, or passive, damping technique must be developed.

In the following, some of the most relevant active damping methods are evaluated to find the correct approach for this application. Most of them are not suitable for the application under consideration, where the resonance frequency is close to the Nyquist frequency.

\section{A. Capacitor current feedback active damping}

This method is based on the implementation of a virtual resistor in parallel with the filter capacitor by the proportional feedback of its current [7]. Due to the delay introduced by the digital implementation of the controller the virtual resistance becomes a virtual impedance, whose real part becomes negative if the resonance frequency is located between $F_{\text {samp }} / 6$ and $F_{\text {samp }} / 2$, leading to stability issues. As the resonant frequency is always located between those two values for the application under consideration, stability problems are avoided by changing the feedback sign. Even though it is an effective option for the system under study, it requires additional current sensors. For this reason, other alternatives will be explored.

\section{B. Capacitor voltage feedback active damping}

Another way to introduce a virtual resistor without additional sensors is to use the existing voltage measured at the capacitor for grid synchronization. The derivative of the voltage capacitor is used as an estimation of its current. The estimated current is then used to perform the proportional feedback [8]. Due to the phase lag introduced by the digital controller and the power converter, $-270^{\circ}$ close to the Nyquist frequency, and the deformation created by the discretization of the derivative controller close to the Nyquist frequency, this option is not feasible for this application. The derivative action becomes a proportional action close to the Nyquist frequency [9]. Due to these facts, the lead-lag compensator as an alternative for the derivative controller is not an appropriate choice either.

\section{Filter-based active damping}

In this option a filter is inserted, to achieve the active damping compensation, either in the feedback path of the chosen system variable or in the controller path. The filter purpose, from
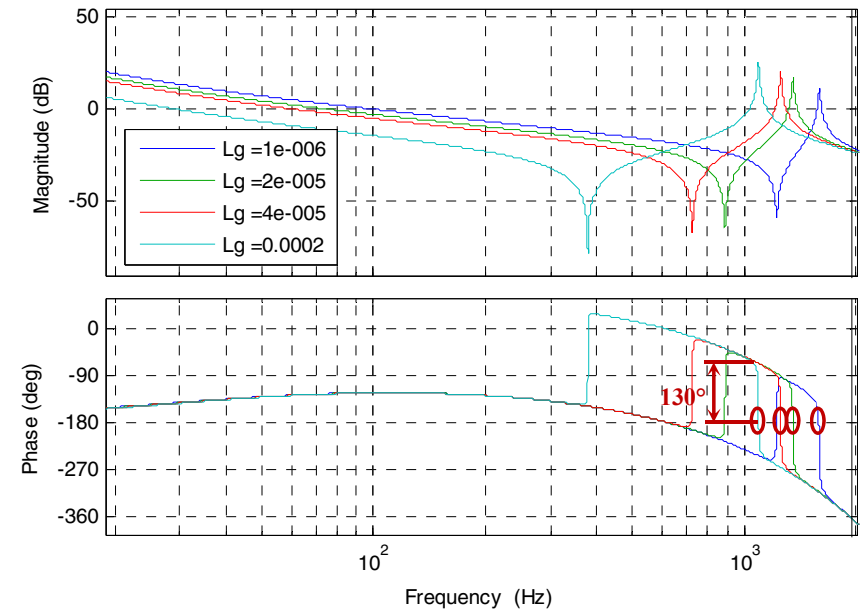

Fig. 6. Open loop Bode plot of the converter current control for a wide range of grid inductance values.

a Bode point of view, is to modify, in the open loop, the phase or the magnitude to avoid $-180 \mathrm{~dB}$ crossings when the magnitude is greater than $0 \mathrm{~dB}$. To avoid such instability in the system either the -180 crossings can be displaced to lower frequencies, where the magnitude is below $0 \mathrm{~dB}$ or the positive resonance peak attenuated. It must be kept in mind that if stability is guaranteed for the worst case with a grid inductance of $200 \mu \mathrm{H}$ $(\mathrm{SCR}=1.9)$, the stable operation would be guaranteed for the most stringent operating conditions.

Different digital filters can be used to attain stability by following this basic principle, mainly notch filters, lead-lag filters and low pass filters. An effective option is the use of notch filters, able to cancel the resonant peak [9]. Unfortunately, this filtering option requires a precise knowledge of the resonance frequency and it might suffer strong variations, particularly in weak grids. Low pass filters and lead-lag filters are an alternative to the notch filters [9]. The lead-lag filters are an appropriate solution for lower ratios of the resonant frequency to the Nyquist frequency [9] as they can introduce enough phase to avoid the $-180^{\circ}$ crossings. However, this is not the case covered in this work. Low pass filters can be used to gain stability by either modifying the magnitude or the phase. A low pass filter that introduces enough magnitude attenuation as to stabilize the system might not be a feasible solution for the system under consideration, as it will reduce the stability margins of the current loop. It must be kept in mind that the resonant frequency and the current loop bandwidth are located that close that a low pass filter able to attenuate the resonant frequency will highly reduce the controller bandwidth. In contrast, $-180^{\circ}$ crossings can be displaced to lower frequencies by introducing a phase lag high enough as to ensure stability for the worst case under consideration. This later approach is the one developed in this work for the parallel interleaved voltage source power converter.

In the next section a comprehensive approach for the design of the active damping low pass filter is provided along with the stability analysis. 


\section{Proposed Active Damping: Design AND Stability ANALYSIS}

As stated above, a low pass digital filter that introduces enough phase lag as to stabilize the system is the option chosen in this work. Through the system modeling and the stability analysis performed, the required phase delay in order to stabilize the system can be calculated. The required phase delay can be deduced from the open loop Bode plot by analyzing the phase delay that must be introduced to avoid negative crossings with $\pm(2 \mathrm{k}-1) \times 180^{\circ}$ at the lowest resonance frequency, which corresponds to the greatest grid inductance considered at the point of common coupling. This design is robust, as the resonance frequency exhibits an asymptotic behavior when the grid inductance is increased, Fig. 3.

The converter current control loop depicted in Fig.1 is the one represented in Fig. 7, including the active damping low pass filter for the stabilization purpose. The digital filter is inserted in the current control loop, so no additional sensors are required.

To avoid instability for the values provided in Table I, according to Fig. 6, a minimum phase delay of 130 degrees has to be introduced at $1 \mathrm{kHz}$, corresponding to the upper bound considered for the grid inductance. A maximum phase delay of 250 degrees can be introduced at $1.6 \mathrm{kHz}$ in order to avoid such negative crossings with $-540^{\circ}$. To achieve the required phase delay the implementation of a second order filter seems to be a suitable solution. However, such filter would have its cut off frequency at a low frequency in order to achieve the required phase lag, causing an unacceptable reduction of the current control loop phase margin. For this reason, a third order filter is chosen as the best option to achieve stability.

By selecting an appropriate filter, a compromise between magnitude flatness and small phase delay within the controller bandwidth can be achieved. A Butterworth filter is selected for the active damping purpose. The filter cut-off frequency is set to $800 \mathrm{~Hz}$, introducing a phase lag of $-160^{\circ}$ at a frequency of $1 \mathrm{kHz}$, shown in Fig.8. The phase margin is reduced by $11^{\circ}$ with the proposed filter, while the bandwidth remains unaffected. This reduction of the phase margin can be easily recovered by modifying the PI controller parameters. As the grid inductance is increased the controller bandwidth is decreased, a readjustment of the controller proportional gain would help to improve the system dynamic response.

As shown in Fig. 9, thanks to the third order filter the $-180^{\circ}$ crossings are produced at a lower frequency, where the magnitude is below $0 \mathrm{~dB}$. The crossings with $-540^{\circ}$ are also well below the $0 \mathrm{~dB}$ gain. It can be concluded that stability is guaranteed without penalizing the dynamic response of the current control. Fig. 10 shows the pole zero map for the close loop transfer function of the converter current control for different values of the grid inductance, and thus, different values for the resonance frequency, both when the active damping filter is enabled and disabled. The transfer function of the converter current to the converter voltage is discretized with the $\mathrm{ZOH}$ transformation and the sampling and calculation sample time delay is also taken into account, Fig. 7. The stabilizing effect of the third order filter is thus verified since the unstable closed loop poles are moved towards the stability region even for the weakest grid conditions, validating the study performed in the s- domain by means of the Nyquist stability criteria.

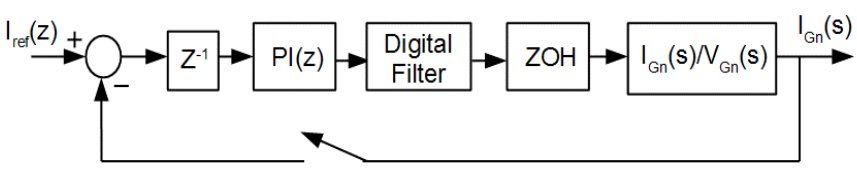

Fig. 7. Complete current control loop.

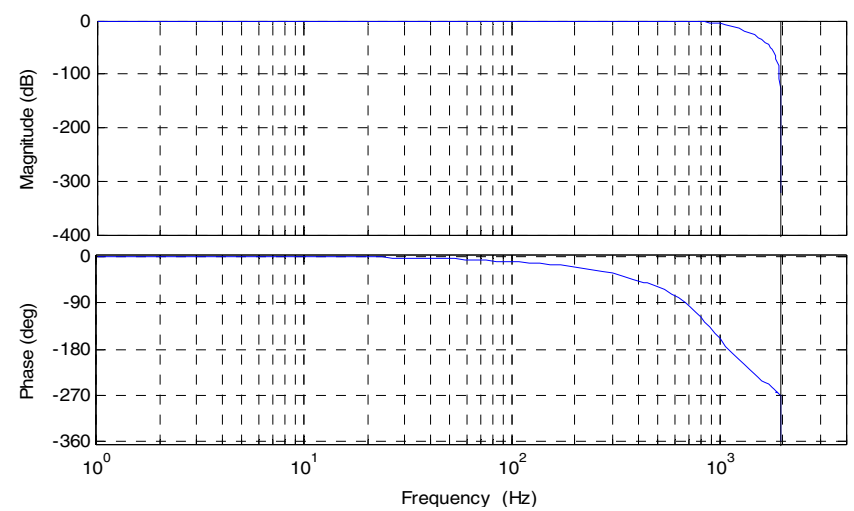

Fig. 8. Third order Butterworth filter.
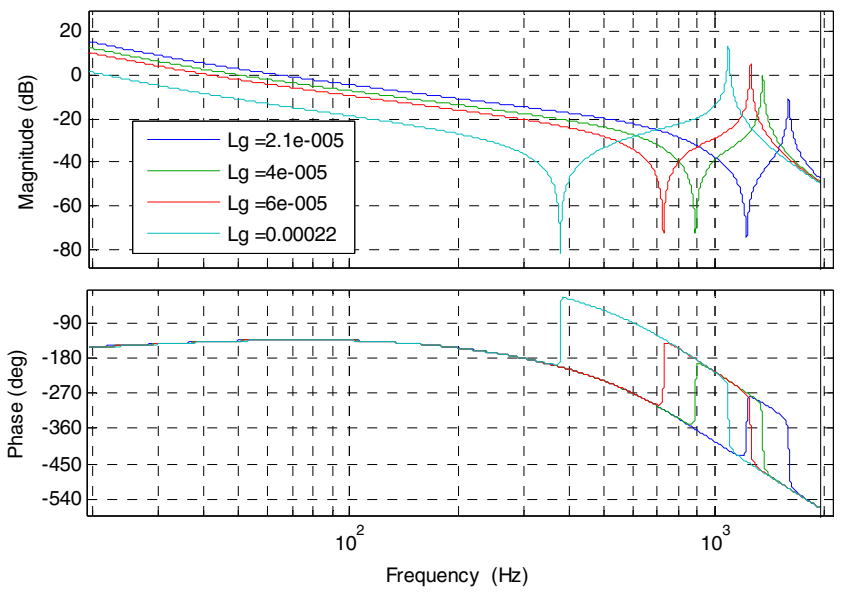

Fig. 9. Bode plot of the converter current control with the proposed active damping strategy.

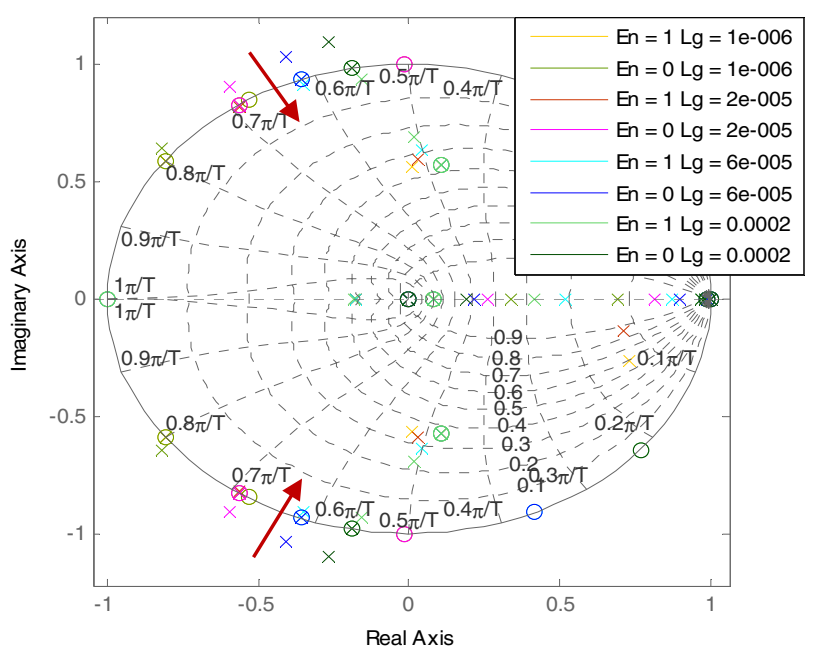

Fig. 10. Pole-zero map of the discrete system for different values of $L_{g}$ with $(E n=1)$ and without $(E n=0)$ the active damping third order filter. 


\section{VALidATion OF THE STRATEGY}

To test the performance of the proposed active damping strategy, simulations using Matlab SimPowerSystems toolbox have been carried out. The simulated system is formed by two parallel 2MW power converters with the specifications of Table I, to create a $4 \mathrm{MW}$ power conversion structure. Both power converters are coupled by single phase interphase transformers (IPTs) with a $90 \mu \mathrm{H}$ magnetic inductance, in order to limit the circulating currents, and a negligible leakage inductance, so they do not affect the normal current component. The modulation used is the space vector modulation, SVPWM7 [22]. The control loop is programmed in an S-Function, taking as inputs the measured converter currents and the capacitor voltage and is executed twice per carrier cycle, performing asymmetrical sampling. The output of the control function are the gate signals, Fig.1, considering a dead time of $4 \mu \mathrm{s}$. The parasitic series resistance of the transformer, converter inductance and filter capacitor are considered to be $0.15 \mathrm{~m} \Omega, 0.1 \mathrm{~m} \Omega$ and $1 \mathrm{~m} \Omega$ respectively. A grid inductance value of $2 \mu \mathrm{H}$, SCR of 190 if only a power converter is connected to the grid, is considered for the analysis presented in this section.

Fig. 11 shows the grid current waveform, at half of the converter rated power and unity power factor, as the active damping third order filter is connected at $1.1 \mathrm{~s}$. To check the effectiveness of the active damping strategy, initially, the active damping is disabled and the power converter is unstable as it can be deduced from the initial current waveform. When the active damping strategy is enabled, the system is stabilized, and the current is controlled to its desired value and power factor, proving the effectiveness of the strategy. This simulation verifies the results obtained in Fig. 10.

The robustness of the solution against grid inductance variations is tested by abruptly modifying the grid inductance seen by the power converter, Fig. 12. The effective grid inductance changes with the number of power converters connected to the grid. Initially, the power converter is connected to a grid inductance of $1 \mu \mathrm{H}$, or SCR of 380 . At time 0.4 seconds, the grid inductance is instantaneously increased to $38 \mu \mathrm{H}$, corresponding to a weak grid with a SCR of 10 . The active damping strategy proves its robustness, as it is able to maintain the system stable operation in two highly different grid inductance situations. Moreover, the system offers a fast dynamic response in the new operating conditions.

The stable operation is not the only requirement that must be met by the power converter. The current harmonic content must comply with the limits imposed by the grid codes. As the fulfillment of such limits is strongly affected by the damping introduced, in the following, the grid current harmonic content is compared to the harmonic limits imposed by the BDEW [3], Fig. 13. The grid inductance selected for the simulation is $2 \mu \mathrm{H}$, corresponding to a short circuit ratio of 190 . For this value the corresponding harmonic limits are calculated. The THD of the phase current is $1.51 \%$, but the grid current does not fulfill the grid code requirements as, at the resonance frequency, a great magnitude peak exists in the magnitude Bode plot even though the system has been stabilized, Fig.9. It should be noted that the commutation frequency is set to $1950 \mathrm{~Hz}$ but those harmonics are recirculated and limited by the interphase transformer, and thus they do not appear in the grid current.

To reduce the peak at the resonance frequency a small resistance in series with the filter capacitor is added.

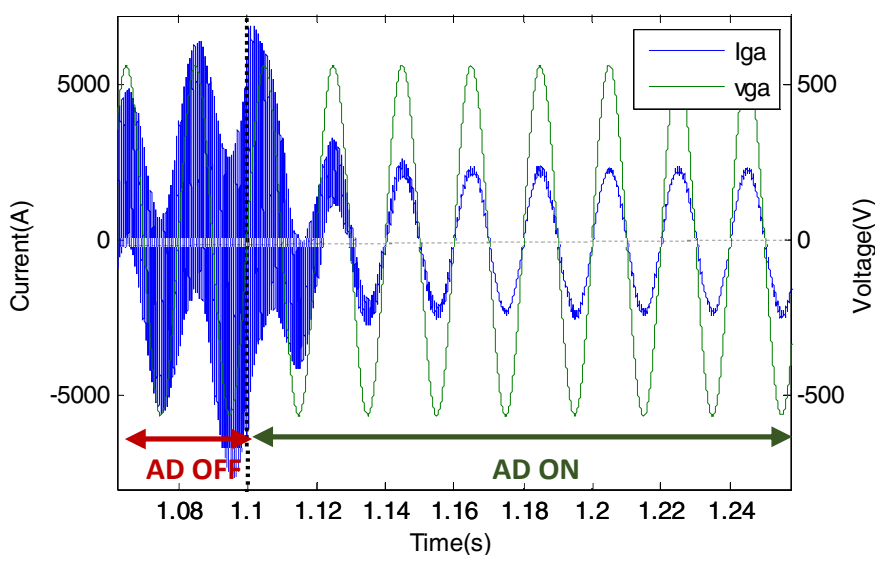

Fig. 11. Evolution of the grid current for phase $a$ as the active damping (AD) third order filter is connected.

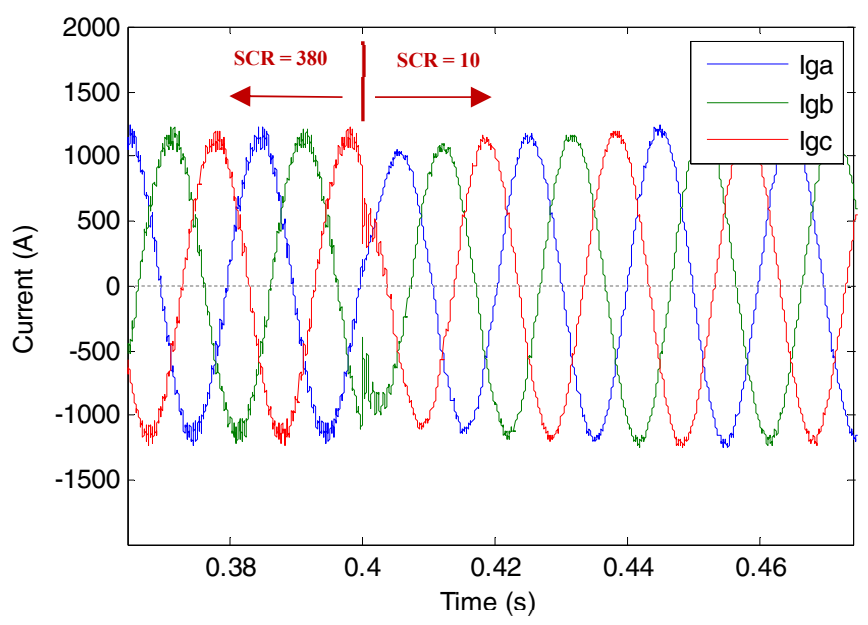

Fig. 12. Three phase grid current as the grid inductance is suddenly changed from a value of $1 \mu \mathrm{H}$, SCR of 380 , to a value of $38 \mu \mathrm{H}$, SCR of 10 .

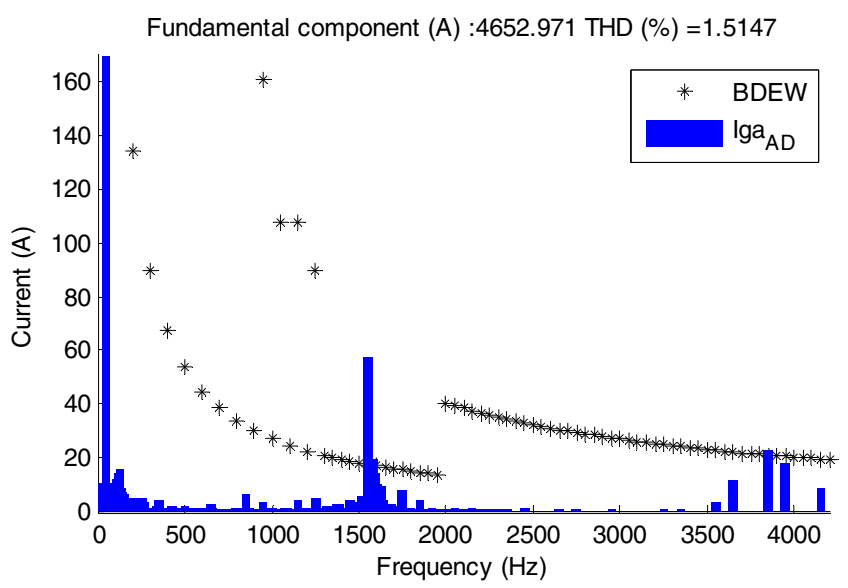

Fig. 13. Grid current harmonic content with $L g$ of $2 \mu \mathrm{H}$ 


\section{COMPARISON BETWEen THE ACTIVELY AND PASSIVELY DAMPED SYSTEMS}

In order to compare the actively damped and the passively damped systems in terms of grid current harmonic content and power losses, an optimized design of the damping must be developed for both systems.

In the actively damped system the stability is guaranteed by the low pass filter. The third order digital filter is adjusted in order to introduce the required phase delay at the lowest resonance frequency. The filter cut-off frequency is set to the lowest plausible value, so an extra damping can be provided to the resonant poles through an attenuation of the magnitude at the resonance frequency. Nevertheless, its cut-off frequency is bounded, as a lower cut-off frequency reduces the stability margins of the current loop through the reduction of the phase margin, Fig. 8. Once the active damping filter is adjusted, the grid current harmonic compliance is verified. If the requirements are not met, a resistor in series with the filter capacitor is added, the value of the resistor is increased until the grid current harmonic content limits imposed by the corresponding grid code are satisfied. The grid code taken as reference [3] imposes different limits depending on the SCR, so the required resistor is calculated for every grid inductance considered.

In the actively damped system, the stability and the grid code compliance are determined by the attenuation introduced by the capacitor series resistor. In a first step, the resistor required to stabilize the system for the greatest grid inductance is calculated. In a second step, the harmonic grid current content is verified for every grid inductance considered. If the requirements are not met, the series resistor is increased until they are fulfilled for every case considered.

In Fig. 14 the required resistances calculated by the application of the previous design procedure are plotted. As expected, the required resistance for the passively damped (PD) system can be 2.4 times greater than for the actively damped (AD). The graph contains useful information for the design procedure. If the worst case effective grid inductance is known, the appropriate resistor can be selected in order to guarantee the stable operation and the harmonic grid code compliance. As a consequence of the reduction in the required resistance for the AD system, the power losses are reduced with respect to the PD system, Fig. 15. As expected, the design procedure guarantees the fulfillment of the harmonic current limits imposed by the BDEW, Fig. 16.

In addition to the benefits previously described, the proposed active damping strategy offers an interesting feature. In existing renewable energy generating plants, if the power connected at the point of common coupling is increased, stability issues can arise, as the effective grid inductance seen by each power converter grows. The proposed active damping strategy allows to solve the stability issues by a modification in the software, through the inclusion of the digital low pass filter, without modifying the damping resistor. As a consequence the active damping method investigated in this paper could be a cost effective solution for existing grid-connected power converters to solve stability problems due to the robustness of the solution.

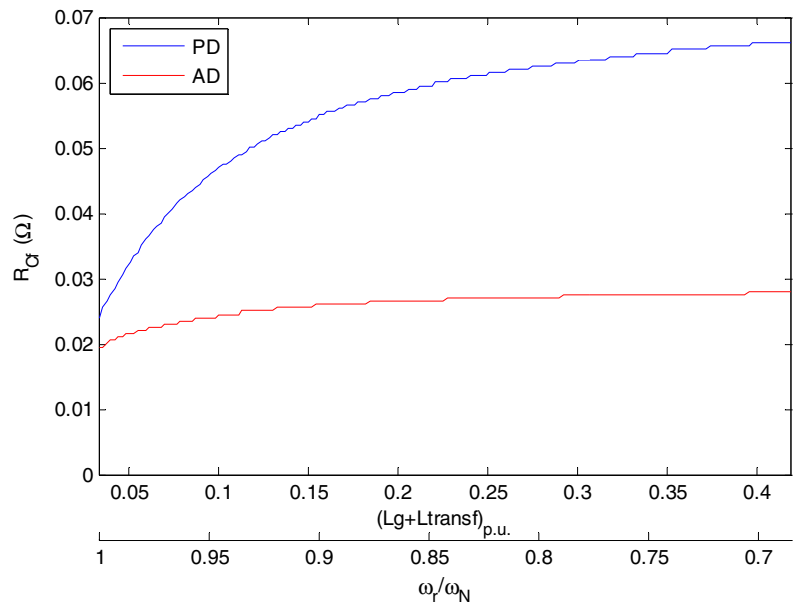

Fig. 14. Required resistor for the system with active damping (AD) and the system with no active damping (PD) as the grid inductance is increased and thus the resonance frequency modified.

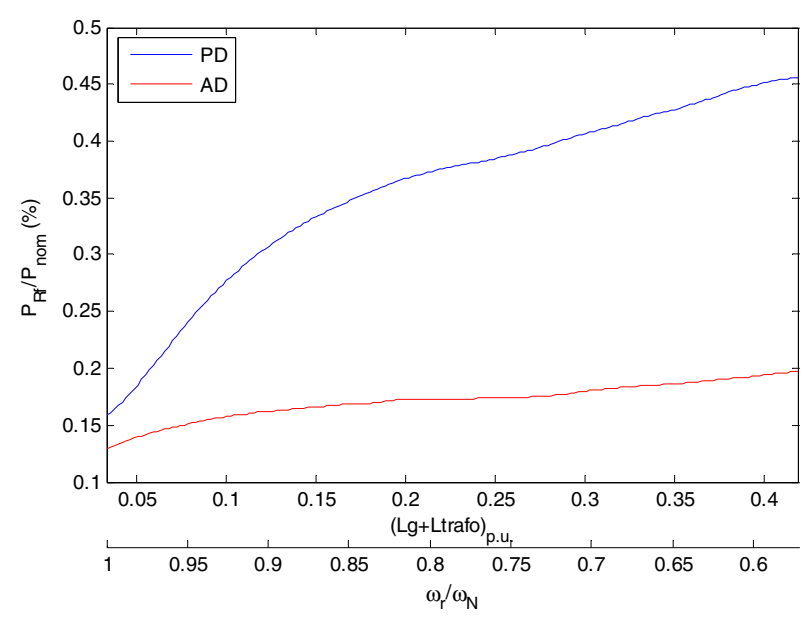

Fig. 15. Power losses at the resistive element added in series with the filter capacitor for de $\mathrm{AD}$ and $\mathrm{PD}$ systems.

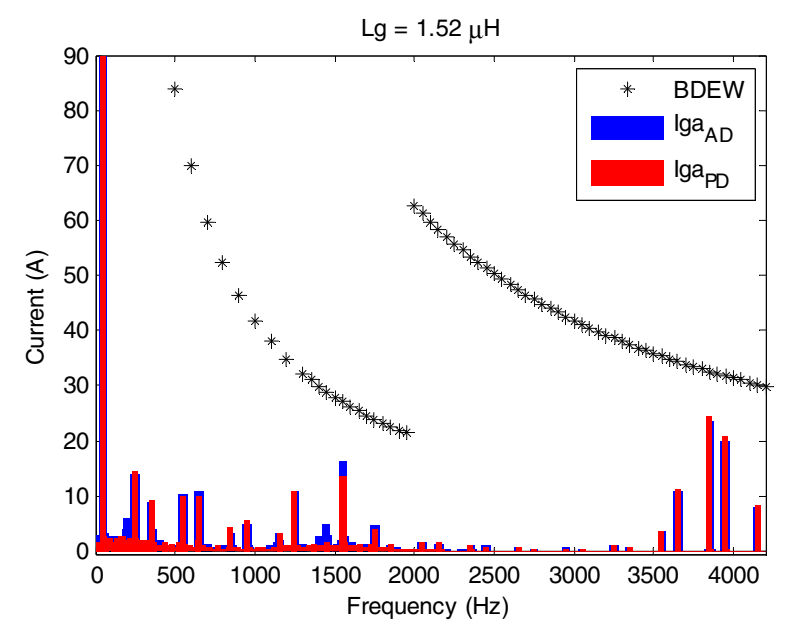

Fig. 16. Grid current harmonic content for the AD and PD systems compared with the limits imposed by [3] for a SCR of 246.

\section{CONCLUSIONS}

In this work the active damping of the LCL filter of two interleaved power converters has been discussed. The proximity of the resonance frequency to the Nyquist frequency requires a 
precise examination of the digitalization of the controller. Three different active damping approaches have been studied. The current capacitor feedback active damping is discarded as it requires additional sensors. The derivative of the capacitor, commonly used to avoid the extra sensor, is not appropriate for the system under study due to the proximity of the Nyquist frequency to the LCL resonance frequency. An active damping approach based on a low pass digital filter has been developed. The proposed active damping does not require additional sensors and can be easily tuned for different systems by analyzing the Bode diagrams. The stability has been tested for different grid inductance values, allowing the stable operation under stringent conditions. As the stability is guaranteed through a phase lag technique, only a small resistor has to be added in order to meet the most demanding grid code current harmonic limits. The harmonic content requirements are met with reduced power losses, compared to the passively damped case. Additionally, the active damping approach presented in this work is a cost effective option for existing grid-connected power converters with stability issues due to changes in the effective grid impedance for which they were designed.

\section{ACKNOWLEDGMENT}

The authors gratefully acknowledge INGETEAM POWER TECHNOLOGY and the Spanish Ministry of Economy and Competitiveness under grant DPI2013-42853-R for its financial and ongoing support. This work was partially funded by the Public University of Navarre through a doctoral scholarship.

\section{REFERENCES}

[1] "Power Generation Systems Connected to the Low-Voltage Distribution Network-Technical Minimum Requirements for the Connection to and Parallel Operation With Low-Voltage Distribution Networks," 2010.

[2] "IEEE Application Guide for IEEE Std 1547, IEEE Standard for Interconnecting Distributed Resources With Electric Power Systems." IEEE Standard 1547.2-2008, 2009.

[3] "Technical Guideline: Generating Plants Connected to the MediumVoltage Network.” BDEW, Berlin, Germany, 2008.

[4] M. Liserre, F. Blaabjerg, and S. Hansen, "Design and Control of an LCL Filter-Based Three-Phase Active Rectifier," IEEE Trans. Ind. Appl., vol. 41, no. 5, pp. 1281-1291, 2005.

[5] M. Routimo and H. Tuusa, "LCL type supply filter for active power filter Comparison of an active and a passive method for resonance damping," PESC Rec. - IEEE Annu. Power Electron. Spec. Conf., pp. 2939-2945, 2007.

[6] R. Beres, X. Wang, F. Blaabjerg, M. Liserre, and C. Bak, "A Review of Passive Power Filters for Three-Phase Grid Connected Voltage-Source Converters," Emerg. Sel. Top. Power Electron. IEEE J., vol. PP, no. 99, p. $1,2015$.

[7] X. Wang, F. Blaabjerg, and P. C. Loh, "Virtual RC Damping of LCL -
Filtered Voltage Source Harmonic Compensation," IEEE Trans. Power Deliv., vol. 30, no. 9, pp. 4726-4737, 2015.

[8] R. Pena-Alzola, M. Liserre, F. Blaabjerg, R. Sebastian, J. Dannehl, and F. W. Fuchs, "Systematic Design of the Lead-Lag Network Method for Active Damping in LCL-Filter Based Three Phase Converters," Ind. Informatics, IEEE Trans., vol. 10, no. 1, pp. 43-52, 2014.

[9] J. Dannehl, M. Liserre, and F. W. Fuchs, "Filter-based active damping of voltage source converters with LCL filter," IEEE Trans. Ind. Electron., vol. 58, no. 8, pp. 3623-3633, 2011.

[10] C. Zou, S. Member, B. Liu, S. Duan, R. Li, and S. Member, "Influence of Delay on System Stability and Delay Optimization of Grid-Connected Inverters With LCL Filter," IEEE Trans. Ind. Informatics, vol. 10, no. 3, pp. 1775-1784, 2014.

[11] B. V. Yaramasu, B. Wu, P. C. Sen, S. Kouro, and M. Narimani, "HighPower Wind Energy Conversion Systems : State-of-the-Art and Emerging Technologies," Proc. IEEE, vol. 103, no. 5, 2015.

[12] J. S. Siva Prasad and G. Narayanan, "Minimization of grid current distortion in parallel-connected converters through carrier interleaving," IEEE Trans. Ind. Electron., vol. 61, no. 1, pp. 76-91, 2014.

[13] D. Zhang, F. Wang, R. Burgos, R. Lai, and D. Boroyevich, "Impact of Interleaving on AC Passive Components of Paralleled Three-Phase Voltage-Source Converters," vol. 46, no. 3, pp. 1042-1054, 2010.

[14] R. S. Zhang, C. Wang, Z. Tan, and H. Weng, "Cross current control for power converter system," US 7852643 B2, 2010.

[15] E. Labouré, A. Cunière, T. A. Meynard, and F. Forest, “A Theoretical Approach to InterCell Transformers , Application to Interleaved Converters," vol. 23, no. 1, pp. 464-474, 2008.

[16] G. Gohil, L. Bede, R. Teodorescu, T. Kerekes, and F. Blaabjerg, "Integrated Inductor for Interleaved Operation of Two Parallel Three-phase Voltage Source Converters," in EPE'15 ECCE Europe, 2015, pp. 6-9.

[17] D. Zhang, F. Wang, R. Burgos, R. Lai, and D. Boroyevich, "Impact of interleaving on $\mathrm{AC}$ passive components of paralleled three-phase voltagesource converters," IEEE Trans. Ind. Appl., vol. 46, no. 3, pp. 1042-1054, 2010.

[18] S. K. T. Miller, T. Beechner, and J. Sun, "A comprehensive study of harmonic cancellation effects in interleaved three-phase VSCs," PESC Rec. - IEEE Annu. Power Electron. Spec. Conf., pp. 29-35, 2007.

[19] J. L. Agorreta, M. Borrega, J. López, and L. Marroyo, "Modeling and control of N-paralleled grid-connected inverters with LCL filter coupled due to grid impedance in PV plants," IEEE Trans. Power Electron., vol. 26, no. 3, pp. 770-785, 2011.

[20] D. G. Holmes, B. P. Mcgrath, C. S. Engineering, and W. Road, "Opportunities for Harmonic Cancellation with Carrier Based PWM for Two-Level and Multi-Level Cascaded Inverters," 1999.

[21] V. Blasko and V. Kaura, "A novel control to actively damp resonance in input LC filter of a three-phase voltage source converter," IEEE Trans. Ind. Appl., vol. 33, no. 2, pp. 542-550, 1997.

[22] H. W. van der Broeck, H. C. Skudelny, and G. V. Stanke, "Analysis and Realization of a Pulsewidth Modulator Based on Voltage Space Vectors," IEEE Trans. Ind. Appl., vol. 24, no. 1, pp. 142-150, 1988. 